\title{
Using algorithmic trading to analyze short term profitability of Bitcoin
}

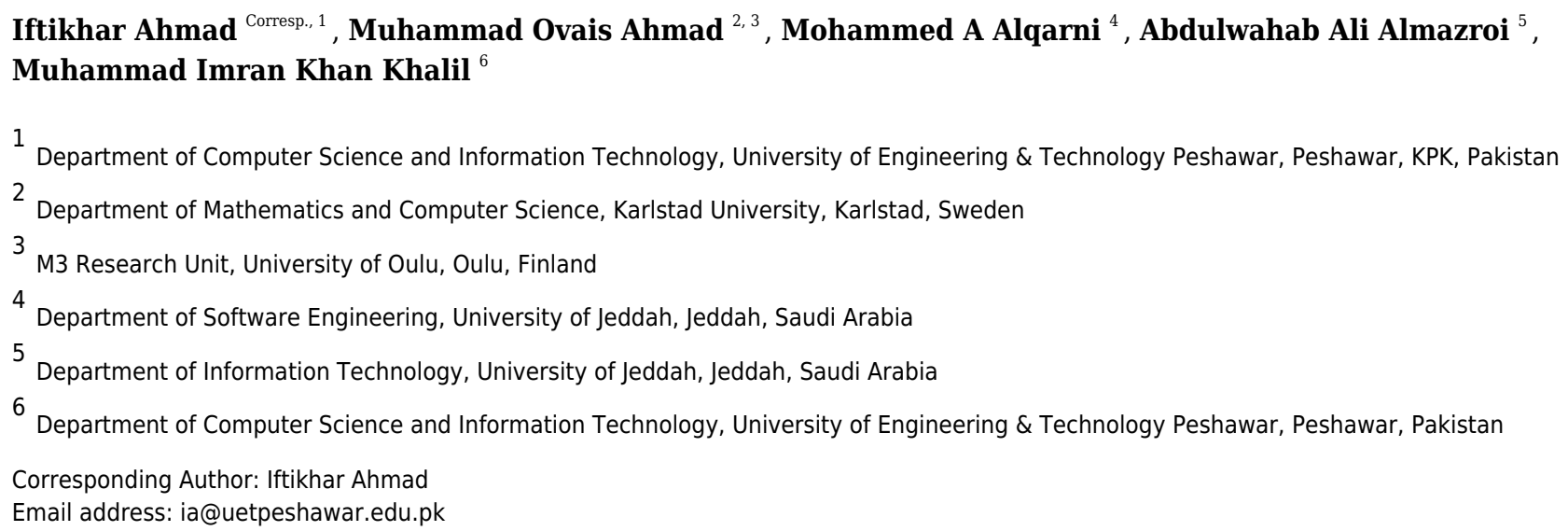

Cryptocurrencies such as Bitcoin (BTC) have seen a surge in value in the recent past and appeared as a useful investment opportunity for traders. However, their short term profitability using algorithmic trading strategies remains unanswered. In this work, we focus on the short term profitability of BTC against Euro and Yens for an eight year period using 7 trading algorithms over trading periods of length 15 and 30 days. We use the classical buy and hold (BH) as a benchmark strategy. Rather surprisingly, we found that on average, Yen is more profitable than BTC and Euro, however the answer also depends on the choice of algorithm. Reservation price algorithms result in $7.5 \%$ and $10 \%$ of average returns over 15 and 30 days respectively which is the highest for all the algorithms for the three assets. For BTC, all algorithms outperform the BH strategy. We also analyze the effect of transaction fee on the profitability of algorithms for BTC and observe that for trading period of length 15 no trading strategy is profitable for BTC. For trading period of length 30 , only two strategies are profitable. 


\title{
Using Algorithmic Trading to Analyze Short Term Profitability of Bitcoin
}

3 Iftikhar Ahmad ${ }^{1}$, Muhammad Ovais Ahmad ${ }^{2,3}$, Mohammed A. Alqarni ${ }^{4}$, Abdulwahab Ali Almazroi ${ }^{5}$, and Muhammad Imran Khan Khalii ${ }^{1}$

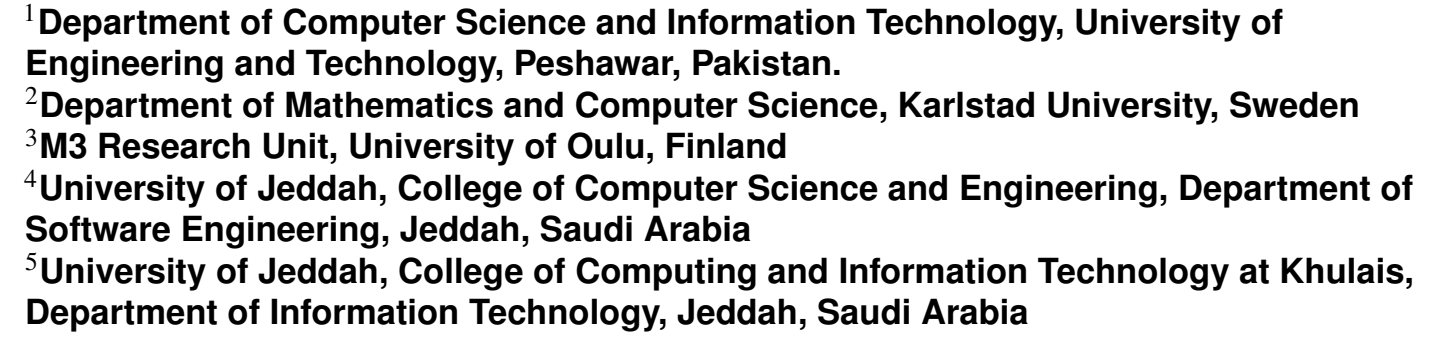

\begin{abstract}
Cryptocurrencies such as Bitcoin $(B T C)$ have seen a surge in value in the recent past and appeared as a useful investment opportunity for traders. However, their short term profitability using algorithmic trading strategies remains unanswered. In this work, we focus on the short term profitability of $B T C$ against Euro and Yens for an eight year period using 7 trading algorithms over trading periods of length 15 and 30 days. We use the classical buy and hold $(B H)$ as a benchmark strategy. Rather surprisingly, we found that on average, Yen is more profitable than BTC and Euro, however the answer also depends on the choice of algorithm. Reservation price algorithms result in $7.5 \%$ and $10 \%$ of average returns over 15 and 30 days respectively which is the highest for all the algorithms for the three assets. For $B T C$, all algorithms outperform the $B H$ strategy. We also analyze the effect of transaction fee on the profitability of algorithms for $B T C$ and observe that for trading period of length 15 no trading strategy is profitable for BTC. For trading period of length 30 , only two strategies are profitable.
\end{abstract}

\section{INTRODUCTION}

Cryptocurrencies have seen a surge in the recent past. Researchers and investors alike have focused on the growth and evolution of cryptocurrencies like Bitcoin (BTC), Etherum, and Litecoin etc. Moore (2013) attributed three main factors that contributed towards the rise and adaptation of bitcoins. First, higher profit margins, maintained by credit card agencies for using their platforms has resulted in dis-satisfied customers. The customers are thus lured to use $B T C$, which promises extremely low transaction fee. Second, the anonymity that is offered by the bitcoins. Bitcoins offer the possibility of conducting transactions using pseudonyms and thus omitting the need of using real names. Third is the decentralization of the bitcoin that protects against inflation. Over time, $B T C$ has become one of the choice currencies for online payment and beside others is accepted by tech-giants like Amazon, Apple, Microsoft, and Paypal etc. The introduction of cryptocurrencies provided a new investment domain for the investors, and became a credible investment vehicle (Brière et al., 2015).

Bitcoin was introduced by Satoshi Nakamoto in 2008 (Nakamoto et al., 2008). The inventor Satoshi Nakamoto is a pseudonym and the real identity of the person is not known to the world. Bitcoin is a digital currency, i.e., unlike fiat currencies such as dollar, and Euro etc., it does not have any physical denomination, and is present only in digital form. Beside similarities, such as the price regulation based on demand and supply, there are some key differences between fiat and crypto currencies like BTC. For instance, $B T C$ has no centralized authority (like the Federal Reserve) that controls the supply, i.e., $B T C$ and by extension all cryptocurrencies are decentralized by nature. The value of a fiat currency is generally 
dependent on factors such as inflation rate in a country, the interest rates, balance between import and export and monetary policy. In contrast, the value of $B T C$ can be determined by several factors such as transactional demand, media speculation, buzz around the technology, and acceptability etc (Nguyen et al., 2018; Wang and Vergne, 2017). Other differentiating aspects include legality, tangibility, and storage.

The underlying technology of $B T C$ is blockchain. In its simplest form, a blockchain is a distributed append-only ledger formed by the collection of blocks. The append-only nature of the ledger means that transactions once recorded are tempered-proof and cannot be changed/modified in any form. This property is achieved with the help of cryptographic hash functions (Narayanan et al., 2016). The Bitcoin eco-system is based on peer-to-peer network where a large number of computational nodes are connected (not necessarily directly). The peer-to-peer network omits the need of centralized system, instead it uses the concept of "proof-of-work" to validate transactions. For a detailed description of BTC, its underlying technology and applications, the reader is referred to Narayanan et al. (2016).

Algorithmic trading is an important tool used by investors in financial trading markets (Ahmad and Schmidt, 2012). It facilitates investors in investing their wealth in various assets (currencies, bonds, stock shares etc.) by automating the decision making process. A number of algorithms are proposed in the literature for algorithmic trading (Iqbal and Ahmad, 2015; Mohr et al., 2014; Ahmad and Schmidt, 2012; El-Yaniv et al., 2001). The problem is addressed in a wide variety of domains including computer science (Kao and Tate, 1999; El-Yaniv et al., 2001; Mohr et al., 2014), operations research (Schroeder et al., 2018), economics and finance (Coakley et al., 2016; Hsu et al., 2010). These algorithms are based on various assumptions and are designed to optimize a variety of objective functions such as minimizing competitive ratio (Mohr et al., 2014).

Algorithmic trading and technical analysis are also important tools to investigate the market behavior and assess its profitability in the short and long term scenarios (Ahmad and Schmidt, 2012; Coakley et al., 2016). Despite the debate in the literature questioning the effectiveness of technical analysis, there is a plethora of research work based on technical analysis (Coakley et al., 2016; Hsu et al., 2010; Menkhoff and Taylor, 2007). The variety of studies validated the usefulness of technical analysis and its wide spread applicability. However, to the best of our knowledge, there is no work to evaluate the short term profitability of $B T C$ using algorithmic trading and technical analysis. We investigate the short term profitability of BTC against two other major currencies Euro and Yen. More specifically, we consider daily exchange rates of dollar-BTC, dollar-Euro, and dollar-Yen from $1^{\text {st }}$ Jan 2011 to $31^{\text {st }}$ Dec 2018. We investigate the short term profitability (15 and 30 days) as it is a common observation that in the long term BTC has observed significant price movement and is highly profitable.

We consider two categories of algorithms namely reservation price algorithms and moving average based algorithms and consider buy and hold as a benchmark strategy. Our findings are based on the geometric average period return, the effect of transaction fee, the number of buy and sell transactions, the number of completed transactions, and the number of profitable vs. non-profitable transactions. Using buy and hold strategy as our benchmark, we compare the geometric average period returns of the 7 strategies with buy and hold. Rather surprisingly, we found that in short term Yen is more profitable than BTC and Euro, however the answer also depends on the choice of algorithm. Reservation price algorithms result in $7.5 \%$ and $10 \%$ of average returns over 15 and 30 days respectively, which is the highest for all algorithms for the three assets. For $B T C$, all algorithms outperform the $B H$ strategy. After introducing a transaction fee of $4 \%$, we observe that for the trading period of length 15 no trading strategy is profitable for $B T C$, whereas for trading period of length 30 , only two strategies are profitable.

It is important to mention that we do not consider machine learning techniques but instead focus on algorithmic trading strategies which do not rely on past trends and patterns, thus do not need future to follow the patterns of the past. Machine learning based algorithms are presented in the literature, the reader is referred to Uras et al. (2020), Alessandretti et al. (2018), and Żbikowski (2016)

Rest of the paper is organized as follows; In Section 2, we briefly present literature review on the use of experimental evaluation of trading algorithms. In Section 3, we present a set of research questions and the methodology for the extraction of data set. In Section 4, we describe the set of algorithms, followed by the description of the evaluation criterion. Results are presented in Section 5, whereas Section 6 presents conclusion, and directions for future work. 


\section{LITERATURE REVIEW}

Experimental evaluation of trading strategies is an established area of research in Computer Science (Iqbal et al., 2012; Ahmad and Schmidt, 2012; Mohr et al., 2014), and Computational Finance (Brock et al., 1992; Coakley et al., 2016). Ever since the seminal work of Brock et al. (1992) there is a considerable literature devoted to the study of algorithmic trading strategies. The strategies are investigated from different perspectives and for various markets around the world. In the following, we present a brief literature review of the work based on experimental analysis of trading algorithms.

Iqbal et al. (2012) performed an experimental evaluation of DAX30 to answer the question "Can online trading algorithms beat the market?". The authors considered a number of trading algorithms, and compared their performance with classical buy and hold $(B H)$ algorithm over trading periods of various length. They concluded that trading algorithms can beat the market, i.e., a trading algorithm can achieve a better return (profit) than $B H$ algorithm. Ahmad and Schmidt (2012) presented an extensive experimental evaluation of trading algorithms for uni-directional conversion problem (see Mohr et al. (2014) for a definition of uni-directional conversion problem). The authors considered two data sets $D A X 30$ and $S \& P 500$ over a period of 10 years $(2001-2010)$, and compared the performance of various algorithms using average competitive ratio. Unlike, Iqbal et al. (2012), Ahmad and Schmidt (2012) used bootstrapping to avoid data snooping bias.

Coakley et al. (2016) performed a comprehensive analysis of various trading rules for 22 currencies over a period of 19 years. Authors reported evidence of profitability for rules based on classical moving average as well as rules based on Bollinger bands and relative strength index. Jiang et al. (2019) investigated the profitability of trading rules in Chinese stock market. The authors used 19 years daily data from Chinese aggregate market return and confirmed the profitability of trading rules even in the presence of transaction costs. Strobel and Auer (2018) analyzed the diminishing predictive power of fundamental variables and seasonal effects over time. They considered Variable Length Moving Average (VLMA) rules introduced by Brock et al. (1992), and using data set covering 1972 to 2015 concluded that VLMA rules have lost the predictive ability. Chang et al. (2017) used VLMA rules to Taiwanese Stock Exchange (TWSE) and computed excess returns to buy and hold $(B H)$ strategy. The objective of the work was to evaluate the effectiveness of $V L M A$ rules against $B H$. The results confirmed the superiority of $V L M A$ rules against $B H$. The novelty of the work lies in the application of $V L M A$ rules to all individual stock listed on TWSE. Hsu et al. (2016) investigated the profitability of technical trading rules in the forex market by analyzing 30 currencies over a period of 45 years. It is argued that there is a significant evidence of the profitability of technical trading rules for some periods. Likewise, the profitability variations are consistent with the adaptive market hypothesis. Fang et al. (2014) used the technical trading rules of Brock et al. (1992) and out-of-sample tests based on fresh data. They inferred that there is no conclusive evidence to support the predictive ability of these strategies. However, they attributed the lack of predictive ability to potential bias rather than efficient market hypothesis.

Despite the plethora of work dedicated to analyze the profitability of various trading strategies and markets, to the best of our knowledge there is no work that compares the profitability of Bitcoin with various other currencies, and to evaluate the performance of various algorithms on Bitcoin.

\section{RESEARCH QUESTIONS AND DATA SET}

\subsection{Research Questions}

We formulate a set of research questions (RQ), which essentially provide a base for the data analysis. The main objectives of the research questions are to identify the most profitable asset, the most appropriate (profitable) algorithm for various assets, and to analyze the effect of transaction cost on the profitability of various algorithms.

RQ 1. Which asset is the most profitable in terms of geometric average period returns?

RQ 2. Which strategy is the most profitable for each of the assets?

RQ 3. How the number of buy and sell signals vary for $B T C$ ?

RQ 4. What are the number of positive and negative returned transactions for BTC?

RQ 5. How the transaction fee effects the profitability of algorithms? 
Table 1. Summary Statistics of the Dataset, $\sigma=$ Standard Deviation, $\gamma=$ Skewness, $\mathscr{K}=$ Kurtosis, $\rho(k)=k$ th order correlation

\begin{tabular}{llll}
\hline \hline Asset & Bitcoin & Euro & Yen \\
\hline \hline Observations & 2921 & 2086 & 2086 \\
Minimum & 0.0000517 & 0.674 & 75.82 \\
Maximum & 3.448 & 0.817 & 125.62 \\
Mean & 0.099 & 0.818 & 101.94 \\
$\sigma$ & 0.345 & 0.075 & 14.62 \\
$\gamma$ & 6.234 & 0.0703 & -0.475 \\
Std Error of $\gamma$ & 0.0452 & 0.0535 & 0.0535 \\
$\mathscr{K}$ & 45.178 & -1.327 & -1.069 \\
Std Error of $\mathscr{K}$ & 0.0905 & 0.1071 & 0.1071 \\
$\rho(1)$ & 0.9984 & 2.9856 & 375.7 \\
$\rho(2)$ & 0.9969 & 2.9820 & 375.2 \\
$\rho(3)$ & 0.9953 & 2.9785 & 374.7 \\
$\rho(4)$ & 0.9937 & 2.9752 & 374.3 \\
$\rho(5)$ & 0.9922 & 2.9790 & 373.8 \\
$\rho(6)$ & 0.9906 & 2.9684 & 373.4 \\
$\rho(7)$ & 0.9890 & 2.9651 & 372.9 \\
\hline \hline
\end{tabular}

Note that the research questions are not arbitrarily but are instead rooted in the literature. For instance, $R Q 1$ is based on Hsu et al. (2016) who used Japanese yen, German mark/euro, U.K. pound, and Swiss franc as base currency in their study and evaluated the profitability of technical trading rules. Likewise, $R Q 2$ is variant of research question posed in Abbey and Doukas (2012). In Abbey and Doukas (2012) the authors examined if technical trading rules can be profitable for individual traders. In the similar manner, $R Q 5$ is studied by a number of researchers including Hsu et al. (2016) and Ahmad and Schmidt (2012).

\subsection{Data}

We consider the daily closing prices of the following currencies against dollar;

i Bitcoin (BTC)

ii Euro

iii Yen

A single data point represents the amount of currency that can be purchased by spending 1 US\$. The BTC data is obtained from coindesk website ${ }^{1}$ for a period of 8 years starting from 1 Jan 2011 to 31 Dec 2018. The main reason for the selection of the data set is based on the availability of the data. On many websites such as coindesk, USD-BTC data is only available from 18 July 2010, therefore, we select the starting date to be 1 Jan 2011, and in the process data set consists of complete 8 years. Euro and Yen data is obtained from Yahoo! Finance ${ }^{2}$.

Table 1 reports various statistics for the data. For the sake of comparison, we take a holistic view of the whole data set by reporting the statistics for the 8 years (1 Jan 2011-31 Dec 2018).

\section{EXPERIMENTAL SETUP AND METHODOLOGY}

\subsection{Trading Algorithms}

A variety of trading algorithms are proposed in the literature (Mohr et al., 2014; Coakley et al., 2016). In the following we describe a selected set of algorithms that are used in our study. The motivation behind the selection of the algorithms from the literature is rooted in the performance of the algorithms. Studies (Ahmad and Schmidt, 2012; Iqbal et al., 2012, 2019) have shown that reservation algorithm of El-Yaniv et al. (2001) and Iqbal et al. (2019) are the best performing algorithms. Further, in order to make the

\footnotetext{
${ }^{1}$ www.coindesk.com

${ }^{2}$ finance.yahoo.com
} 
comparison meaningful, two widely used techniques from finance namely variable length moving average, and fixed length moving average are also considered.

\subsubsection{Reservation Price Algorithms}

Reservation price algorithm calculates a threshold price and generates a buy signal when the offered exchange rate is less than or equal to the threshold. A sell signal is generated when the price is at least threshold (Iqbal et al., 2012, 2019; Kao and Tate, 1999). A number of reservation price algorithms are presented in the literature (Mohr et al., 2014; Kao and Tate, 1999; Iqbal et al., 2019; El-Yaniv et al., 2001). In the following we present the selected set of reservation price algorithms considered for our study.

El-Yaniv et al. (2001) assumed a priori information about the lower (minimum possible price $m$ ) and upper (maximum possible price $M$ ) bound of prices, and presented a reservation price algorithm. Let $e_{t}$ be the current exchange price. Algorithm 1 provides formal description for El-Yaniv reservation price algorithm for generating buy and sell signals respectively.

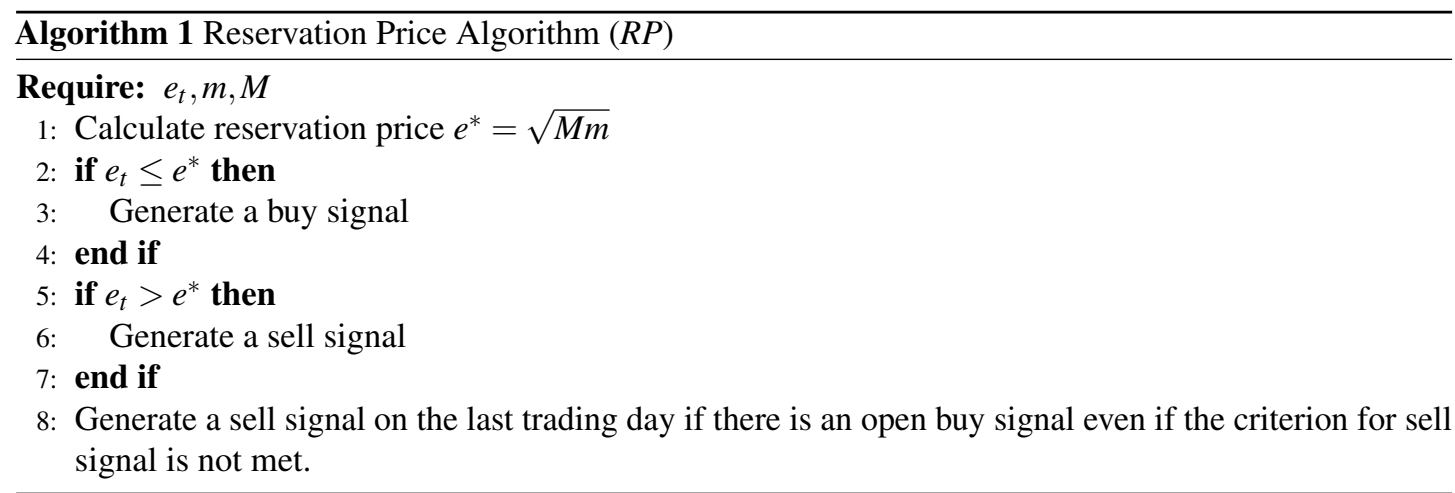

Iqbal et al. (2019) presented a modified version of the reservation price policy of El-Yaniv et al (2001). The authors critiqued the assumption of fixed values of $m$ and $M$ and argued that inter-day price fluctuation is not arbitrary but is instead governed by inter-day price fluctuation function as shown in Eq. 1.

$$
(1-\gamma) e_{t-1} \leq e_{t} \leq(1+\gamma) e_{t-1}
$$

Note that $e_{t}$ is the exchange rate offered on day $t$, and $\left.\gamma \in\right] 0,1[$. The formal description of algorithm is given in Algorithm 2. For detailed working of the algorithm, the reader is referred to Iqbal et al. (2019).

Kao and Tate (1999) presented a reservation price algorithm based on the perceived rank of the offered exchange rate. The perceived exchange rank is calculated based on the current rank $x_{t}$ of the offered exchange rate $e_{t}$ in all the exchange prices observed so far. The formal algorithm is presented in Algorithm 3.

$T$ represents the number of days in a trading period, $\mathscr{L}_{T}(t)$ and $\mathscr{H}_{T}(t)$ are the thresholds for buy and sell signals respectively and are computed as shown in Eq. 2 and Eq. 4 respectively;

$$
\mathscr{L}_{T}(t)= \begin{cases}0 & : t=T \\ \left\lfloor\frac{t+1}{T+1}\left(R_{T}(t+1)-P_{T}(T+1)\right)\right\rfloor & : t<T\end{cases}
$$

Note that $P_{T}(t)$ is the expected difference between the buy and sell prices if the optimal strategy is followed at $t$, and is calculated as shown in Eq. 3 .

$$
\begin{aligned}
& P_{T}(t)= \begin{cases}0 & : t=T \\
P_{T}(t+1)+\frac{\mathscr{L}_{T}(t)}{t}\left(R_{T}(t+1)-P_{T}(t+1)-\frac{T+1}{t+1} \frac{\mathscr{L}_{T}(t)+1}{2}\right) & : t<T\end{cases} \\
& \mathscr{H}_{T}(t)=\left\lceil\frac{t+1}{T+1} R_{T}(t+1)\right\rceil
\end{aligned}
$$



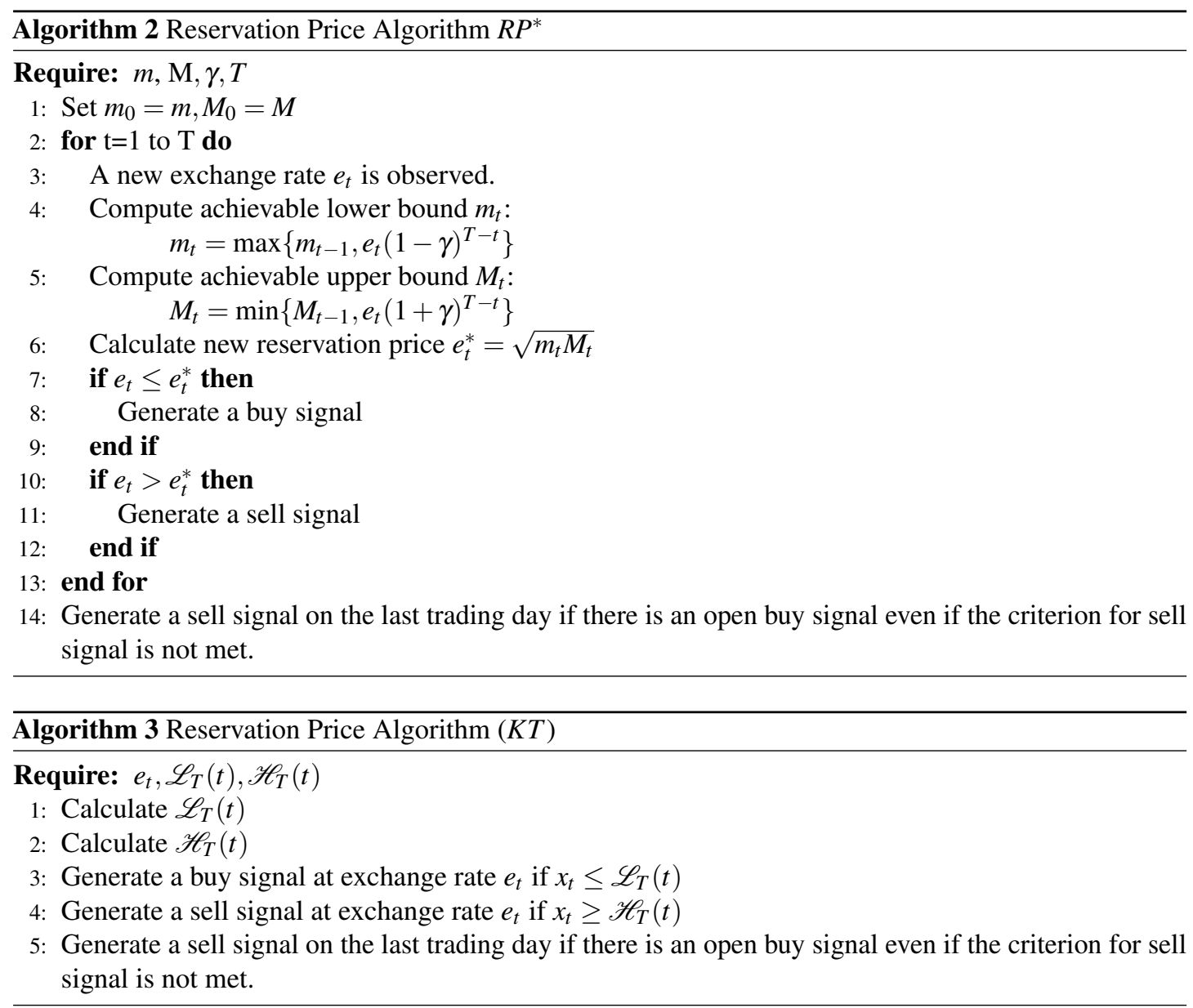

Note that $R_{T}$ is the expected final rank of $e_{t}$ for selling, if an optimal strategy is followed starting from time $t$, and is calculated as given in Eq. 5 .

$$
R_{T}(t)=\frac{\mathscr{H}_{T}(t)-1}{t}\left(R_{t}(t+1)-\frac{T+1}{2(t+1)} \mathscr{H}_{T}(t)\right)+\frac{T+1}{2}
$$

\subsubsection{Moving Average Based Rules}

Moving average $(M A)$ rule is the simplest and popular technical analysis trading rule. The basic idea of $M A$ based rules is to generate buy and sell signals based on the short vs long-term moving averages. More specifically, a buy signal is generated when the short term moving average cuts the long term moving average from below. On the contrary, a sell signal is generated when the short-term moving average cuts the long-term moving average from above. However, in a market the crossing between short-term and long-term moving averages can occur on multiple instances in a short period, resulting in a large number of buy and sell signals (Zhu et al., 2015). The resulting large number of signals are hardly profitable and can force a large transaction fee as well. To avoid this, a minimum threshold called band is introduced. The band introduces a specific percentage difference between the short and long term moving averages in order to generate buy and sell signals.

In the literature two variants of the moving averages, called Variable Length Moving Average (VLMA) and Fixed Length Moving Average (FLMA) are used (Brock et al., 1992; Gunasekarage and Power, 2001; Zhu et al., 2015). Let $\mathscr{A}_{\mathscr{S}}$ be the short term moving average, $\mathscr{A}_{\mathscr{L}}$ be the long term moving average, and $\mathscr{B}$ the band value. Algorithm 4 describes variable length moving average strategy for buy and sell signals.

In $V L M A$ a buy signal is generated when the short term moving average cuts the long term moving average (taking into account the band value) from below, i.e., $\mathscr{A}_{\mathscr{S}}>(1+\mathscr{B}) \mathscr{A}_{\mathscr{L}}$. Likewise, $V L M A$ generates a sell signal when $\mathscr{A}_{\mathscr{S}}<(1-\mathscr{B}) \mathscr{A} \mathscr{L}$. A rule is represented by the combination of three values $\mathscr{S}$ (length of short-term moving average), $\mathscr{L}$ (length of long-term moving average), $\mathscr{B}$ (band). For 


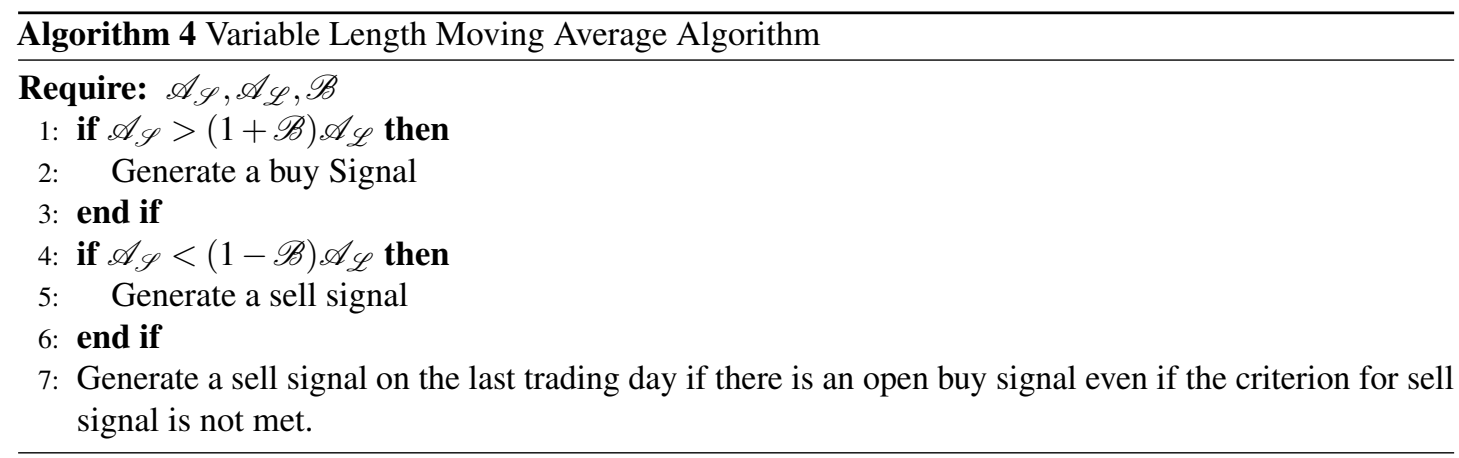

instance, $\operatorname{VLMA}(5,30,0.02)$ represents a rule where short term average is taken over a period of 5 days, long term over a period of 30 days, and the band value is $2 \%$. FLMA works on the same principle as stated in Algorithm 4. However, FLMA differs from the VLMA by introducing a holding period, i.e., once a signal is generated then the position must be held for a fixed number of days. Any signal generated during the holding period is ignored.

\subsubsection{Buy and Hold Strategy}

Buy and hold $(B H)$ is widely used in the literature as a benchmark strategy (Mohr et al., 2014; Chang et al., 2017; Baur et al., 2018), and is therefore used in our study as well. In $B H$ an investor executes the buy transaction on the first day of the investment period and holds the position until the last day $T$. On the last day, a sell transaction is executed to complete the trading. The formal description of buy and sell signals of $B H$ algorithm is given in Algorithm 5.

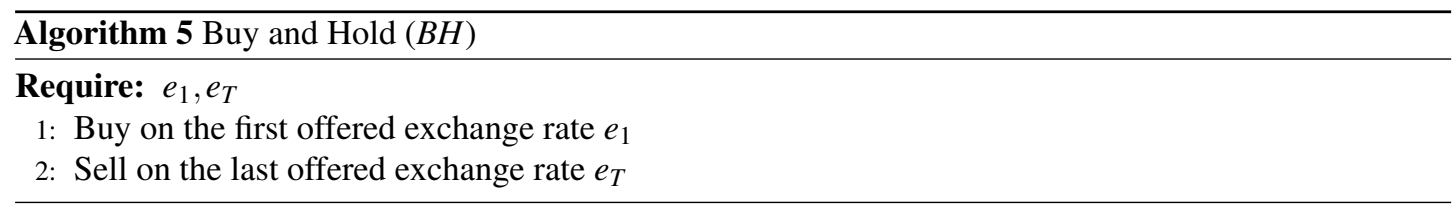

We test the profitability of the algorithms for various parameters and for various durations. We consider short term moving averages over 5 and 10 days, long term moving averages over 15, and 30 days, and band values 0.01 and 0.02 (Brock et al., 1992; Fang et al., 2014). Thus we produce a total of 8 trading rules, 4 each for VLMA and FLMA. Likewise, we consider 15 and 30 days durations for Algorithms 1,2,3, and 5. Thus we have a total of 16 variants of algorithms to evaluate. Table 2 presents a summary of the selected algorithms and their variants.

\subsection{Evaluation Criterion}

We use Geometric Average Trading Period Return (GPR) as our evaluation criterion. GPR is used as an evaluation criterion in a number of works such as Schmidt et al. (2010); Iqbal et al. (2012). Let $D_{0}^{j}$ be the initial amount of dollars at the start of a trading period $j$, and $D_{T}^{j}$ be the final amount of dollars at the end of the trading period $j$. Let, $r_{j}$ be the return of the $j$ th trading period, then $r_{j}=D_{T}^{j} / D_{0}^{j}$. Assuming that there are $P$ trading periods (or trades), we define the geometric average trading period return $G P R(P)$ as;

$$
\operatorname{GPR}(P)=\left(\prod_{i=1}^{P} r_{i}\right)^{1 / P}
$$

Initially we do not consider any transaction fee and report our findings based on zero transaction fee. In Section 5.5, we assess the impact of the transaction fee on the returns by introducing various values of transaction fees. The transaction fees are based on coinbase - one of the popular online services dealing in buying, selling and storage of bitcoins. Coinbase charges a minimum of $1.49 \%$ transaction fee on all transactions. However, the exact value varies based on the mode of payment. For instance, for payment via credit card the transaction fee is $3.99 \%$. We consider a set of transaction fees $T F=\{0,2.0,4.0\}$. We compare the geometric average period returns of the 16 strategies (see Table 2). It is also important to 
Table 2. Summary of the Selected Algorithms and their Variants

\begin{tabular}{|c|c|c|}
\hline S.No & Algorithm & Description \\
\hline 1 & $\operatorname{VLMA}(5,15,0.01)$ & VLMA algorithm with $\mathscr{S}, \mathscr{L}, \mathscr{B}$ values as $5,15,0.01$ \\
\hline 2 & $V \operatorname{LMA}(5,15,0.02)$ & $V L M A$ algorithm with $\mathscr{S}, \mathscr{L}, \mathscr{B}$ values as $5,15,0.02$ \\
\hline 3 & $F \operatorname{LMA}(5,15,0.01)$ & FLMA algorithm with $\mathscr{S}, \mathscr{L}, \mathscr{B}$ values as $5,15,0.01$ \\
\hline 4 & $F \operatorname{LMA}(5,15,0.02)$ & FLMA algorithm with $\mathscr{S}, \mathscr{L}, \mathscr{B}$ values as $5,15,0.02$ \\
\hline 5 & $R P(15)$ & Reservation price algorithm $(R P)$ (El-Yaniv et al., 2001) applied over 15 days \\
\hline 6 & $R P^{*}(15)$ & Update reservation price algorithm $\left(R P^{*}\right)$ (Iqbal et al., 2019) applied over 15 days \\
\hline 7 & $K T(15)$ & Reservation price algorithm (KT) (Kao and Tate, 1999) applied over 15 days \\
\hline 8 & $B H(15)$ & Buy and Hold algorithm applied over 15 days \\
\hline 9 & $V \operatorname{LMA}(10,30,0.01)$ & $V L M A$ algorithm with $\mathscr{S}, \mathscr{L}, \mathscr{B}$ values as $10,30,0.01$ \\
\hline 10 & $V \operatorname{LMA}(10,30,0.02)$ & $V L M A$ algorithm with $\mathscr{S}, \mathscr{L}, \mathscr{B}$ values as $10,30,0.02$ \\
\hline 11 & $F L M A(10,30,0.01)$ & FLMA algorithm with $\mathscr{S}, \mathscr{L}, \mathscr{B}$ values as $10,30,0.01$ \\
\hline 12 & $F L M A(10,30,0.02)$ & $F L M A$ algorithm with $\mathscr{S}, \mathscr{L}, \mathscr{B}$ values as $10,30,0.02$ \\
\hline 13 & $R P(30)$ & Reservation price algorithm $(R P)$ (El-Yaniv et al., 2001) applied over 30 days \\
\hline 14 & $R P^{*}(30)$ & Update reservation price algorithm $\left(R P^{*}\right)$ (Iqbal et al., 2019) applied over 30 days \\
\hline 15 & $K T(30)$ & Reservation price algorithm (KT) (Kao and Tate, 1999) applied over 30 days \\
\hline 16 & $B H(30)$ & Buy and Hold algorithm applied over 30 days \\
\hline
\end{tabular}

mention that returns are only calculated for trading periods when at least one buy transaction is followed by a sell transaction. For situations, where only buy or only sell signals are generated, no returns are taken into account.

\section{RESULTS AND DISCUSSIONS}

In the following, we present our results from various perspectives such as the geometric average trading period returns, the number of buy/sell signals generated and the impact of the transaction fee.

\subsection{Which asset is the most profitable in terms of geometric average period return?}

We calculate geometric average trading period return for each trading rule based on Eq. (6) and report our findings as shown in Table 3. It must be noted that we do not consider any transaction fee in this case. The effect of the transaction fee is discussed later in Section 5.5. It can be seen from the resultant table that the average GPR of the selected assets are 1.016, 1.012, and 1.03 for BTC, Euro and Yen respectively. Although the difference between GPR is not significant, Yen achieved a higher return than BTC, and Euros. A further analysis of the data reflects that the returns are strategy dependent as well. For instance, $R P^{*}(30)$ achieved an GPR of 1.101 over $B T C$ which is the highest returns among all the assets/strategies. Another interesting observation is the number of resultant positive and negative returns. Note that an GPR of at least 1 is termed as a positive return. For BTC, out of 16 strategies, 11 are positive. For Euro and $Y e n$, the corresponding number of positive returns strategies are 16 and 15 respectively.

Comparing the performance of reservation price algorithms $\left(R P, R P^{*}, K T\right)$, and moving average based strategies (VLMA, FLMA) with $B H$, we found that for $B T C$, the returns of all algorithms are superior than corresponding $B H$ strategies. The same trend is observed for Euro except for $K T(30)$ which is inferior to $B H(30)$. All algorithms outperform $B H$ on $Y e n$ as well, except $K T$.

\subsection{Which strategy is the most profitable for each of the assets?}

To answer the question, we analyze Table 3 , and identify the best performing algorithm for each asset. We also consider the trading period length, and summarize the results in Table 4 . We observed that $R P^{*}$ is the best algorithm for $B T C$ achieving a GPR of 1.075 and 1.101 for trading period of length 15 and 30 respectively. For Euro, the corresponding algorithms are $\operatorname{VLMA}(5,15,0.02)$ and $V L M A(10,30,0.02)$ resulting in an average $G P R$ of 1.0432 and 1.021 respectively. $F L M A(5,15,0.02)$ and $F L M A(10,30,0.02)$ are the best performing algorithms for Yen with average GPR of 1.0953 and 1.0952 respectively. It is interesting to note that for each asset, a unique algorithm is adjudicated as the best performing algorithm. Further, analysis reveals that $K T$ and $B H$ are the worst performing algorithms for all the three data sets. For $B T C$, the two algorithms' returns are negative $(<1)$. For Euro, the returns of $K T$ and $B H$ are positive 
Table 3. Geometric Average Trading Period Returns $(G P R)$ of Trading Strategies

\begin{tabular}{llll}
\hline \hline Strategy & Bitcoin & Euro & Yen \\
\hline \hline VLMA $(5,15,0.01)$ & 1.021 & 1.007 & 1.003 \\
$V L M A(5,15,0.02)$ & 1.015 & 1.043 & 1.095 \\
FLMA $(5,15,0.01)$ & 0.986 & 1.007 & 1.005 \\
FLMA $(5,15,0.02)$ & 1.007 & 1.04 & 1.095 \\
$R P(15)$ & 1.062 & 1.009 & 1.01 \\
$R P^{*}(15)$ & 1.075 & 1.01 & 1.011 \\
$K T(15)$ & 0.962 & 1.001 & 1.001 \\
$B H(15)$ & 0.953 & 1.0 & 1.002 \\
$V L M A(10,30,0.01)$ & 1.033 & 1.005 & 1.018 \\
$V L M A(10,30,0.02)$ & 1.041 & 1.021 & 1.095 \\
$F L M A(10,30,0.01)$ & 1.032 & 1.005 & 1.018 \\
$F L M A(10,30,0.02)$ & 1.039 & 1.02 & 1.095 \\
$R P(30)$ & 1.089 & 1.013 & 1.014 \\
$R P^{*}(30)$ & 1.101 & 1.014 & 1.014 \\
$K T(30)$ & 0.929 & 1.0 & 0.998 \\
$B H(30)$ & 0.909 & 1.001 & 1.004 \\
\hline Average GPR & $\mathbf{1 . 0 1 6}$ & $\mathbf{1 . 0 1 2}$ & $\mathbf{1 . 0 3}$ \\
\hline
\end{tabular}

Table 4. Highest $G P R$ achieved for the assets

\begin{tabular}{llll}
\hline \hline Asset & Trading Period & GPR & Algorithm \\
\hline \hline BTC & 15 & 1.075 & $R P^{*}$ \\
BTC & 30 & 1.101 & $R P^{*}$ \\
Euro & 15 & 1.432 & $\operatorname{VLMA}(5,15,0.02)$ \\
Euro & 30 & 1.021 & $\operatorname{VLMA}(10,30,0.02)$ \\
Yen & 15 & 1.0953 & FLMA $(5,15,0.02)$ \\
Yen & 30 & 1.00952 & FLMA $(10,30,0.02)$ \\
\hline
\end{tabular}

(though worst among all), and for Yen the returns are positive except for $K T(30)$ which is marginally less than 1 . On average, $\operatorname{VLMA}(10,30,0.2)$ is the best performing algorithm over all asset by achieving an average $G P R$ of 1.052 which is closely followed by $F L M A(10,30,0.02)$. It is interesting to point out that although $R P$ and $R P^{*}$ assumes apriori information about the lower and upper bounds of future exchange rates, their average performance is inferior to that of VLMA and FLMA.

In order to ensure that the performance of algorithms on BTC is not an anomaly, a statistical $t$-test (paired sample $t$-test) was performed with confidence level of $95 \%(p \leq 0.05)$. The tests were performed on the returns of algorithms for $B T C$ considering 15 and 30 days trading duration. Table 5 and Table 6 summarizes the results of paired t-test for the returns of $B T C$ on various algorithms for 15 and 30 days trading periods. Recall from Table 3 that $R P^{*}$ is the best performing algorithm for BTC. Table 5 confirms that with 95\% confidence the improved performance of $R P^{*}$ over all algorithms (except $V L M A(5,15,0.02)$, and $F \operatorname{LMA}(5,15,0.02))$ is not by chance. For $\operatorname{VLMA}(5,15,0.02)$, and $F \operatorname{LMA}(5,15,0.02)$ the confidence level is still significant (93\% and 94\%). Other than $R P^{*}$, and $R P$ no other algorithm exhibits a significant confidence in the returns over $B T C$. However, except for $F L M A(5,15,0.01)$ and $K T(15)$ all other algorithms have shown the potential to beat the market, i.e., the returns are better (and statistically significant) than $B H$ strategy. For 30 days trading period, the returns of moving average based strategies are statistically significant than $B H$ only (see Table 6 ), whereas $R P^{*}$ achieves statistically significant returns than $R P, K T$, and $B H$ only.

\subsection{How the number of buy and sell signals vary for BTC?}

We record the number of buy and sell signals, as well as the number of completed transactions. A transaction is completed when for a buy signal the corresponding sell transaction occurs. Fig 1 summarizes the number of buy, sell and completed transactions. 
Table 5. Paired sample $t$-test for the returns of BTC with confidence interval of $95 \%$ (15 days trading period)

\begin{tabular}{llllllll}
\hline \hline Algorithms & VLMA $(5,15,0.02)$ & $F L M A(5,15,0.01)$ & $F L M A(5,15,0.02)$ & $R P(15)$ & $R P^{*}(15)$ & $K T(15)$ & $B H(15)$ \\
\hline \hline $\operatorname{VLMA}(5,15,0.01)$ & 0.857 & 0.419 & 0.982 & 0.07 & 0.026 & 0.083 & 0.023 \\
$\operatorname{VLMA}(5,15,0.02)$ & -- & 0.317 & 0.848 & 0.145 & 0.072 & 0.053 & 0.012 \\
$F L M A(5,15,0.01)$ & -- & -- & 0.346 & 0.037 & 0.015 & 0.338 & 0.175 \\
$F L M A(5,15,0.02)$ & -- & -- & -- & 0.127 & 0.061 & 0.089 & 0.041 \\
$R P(15)$ & -- & -- & -- & -- & 0 & 0 & 0 \\
$R P^{*}(15)$ & -- & -- & -- & -- & -- & 0 & 0 \\
$K T(15)$ & -- & -- & -- & -- & -- & -- & 0.944 \\
\hline
\end{tabular}

Table 6. Paired sample $t$-test for the returns of BTC with confidence interval of $95 \%$ (30 days trading period)

\begin{tabular}{|c|c|c|c|c|c|c|c|}
\hline Algorithms & $V L M A(10,30,0.02)$ & $F L M A(10,30,0.01)$ & $F L M A(10,30,0.02)$ & $R P(30)$ & $R P^{*}(30)$ & $K T(30)$ & $B H(30)$ \\
\hline VLMA(10,30,0.01) & 0.541 & 0.425 & 0.574 & 0.559 & 0.393 & 0.054 & 0.043 \\
\hline$V L M A(10,30,0.02)$ & -- & 0.471 & 0.137 & 0.758 & 0.572 & 0.063 & 0.042 \\
\hline FLMA $(10,30,0.01)$ & -- & -- & 0.499 & 0.486 & 0.329 & 0.053 & 0.043 \\
\hline$F L M A(10,30,0.02)$ & -- & -- & -- & 0.73 & 0.546 & 0.065 & 0.43 \\
\hline$R P(30)$ & -- & -- & -- & -- & 0.008 & 0 & 0 \\
\hline$R P^{*}(30)$ & -- & -- & -- & -- & -- & 0 & 0 \\
\hline$K T(30)$ & -- & -- & -- & -- & -- & -- & 0.616 \\
\hline
\end{tabular}

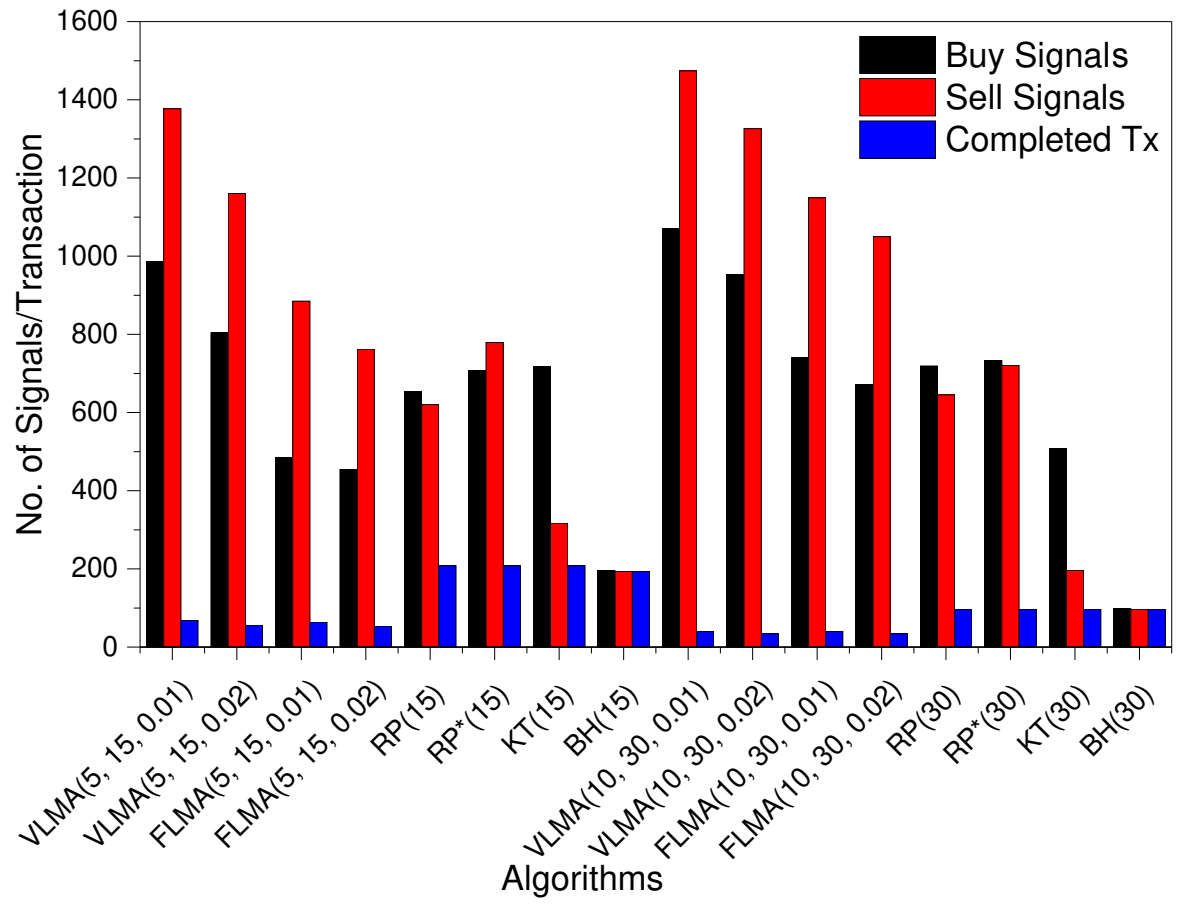

Figure 1. Number of Buy and Sell Signals and Complete Transactions for BTC 
We observed that considering 15 days trading period for BTC, VLMA and FLMA based strategies resulted in $6 \%$ completed transactions. VLMA generates $2 \%$ more buy and sell signals than FLMA. This is logical as VLMA based strategies do not have any holding period and are free to generate a signal if the corresponding criterion is met. For reservation price algorithms, the number of completed transactions are in the range of $27-32 \%$. Buy and hold has the highest number of completed transactions as it does not generate buy and sell signals based on some predefined criterion, but instead buys on the first trading day and sells on the last trading day irrespective of the offered exchange rate. For trading period of length 30 days, the same trend is observed for VLMA and FLMA based strategies.

Our analysis of the data reveals that for VLMA on average $96 \%$ of the buy transactions remains open whereas the corresponding number for the sell signal is $94 \%$. Likewise, the percentage of open buy and sell signals for FLMA are $22 \%$ and $19 \%$ only. For reservation price algorithms, the number of completed transactions are reduced to $14-20 \%$.

\subsection{What are the number of positive and negative returned transactions for $B T C$ ?}

We investigate completed transactions from the perspective of positive vs negative returns for $B T C$. We define a transaction to yield positive returns if the sell price is higher than the buy price, i.e., $r_{j}>1$ where $r_{j}$ represents the returns of the trading period $j$.

For 15 days trading period, we observe that the VLMA and FLMA based strategies have higher percentage $(>50 \%)$ of negative returned transactions. For the reservation price policy, $K T$ has more negative transactions ( $>50 \%$ ) whereas $R P$ and $R P^{*}$ have higher positive transactions. $R P$ has $66.8 \%$, and $R P^{*}$ has 72 positive returned transactions. $B H$ has $45 \%$ positive returned transactions. The worst rate of positive returned transaction is observed for $K T(40 \%)$.

For trading duration of 30 days, rather surprisingly, the percentage of positive returned transaction increased slightly for VLMA, FLMA and $K T$, whereas a reduction is observed for $R P$ and $R P^{*}$. Figure 2 is a graphical summary of the positive and negative returned transaction for BTC.

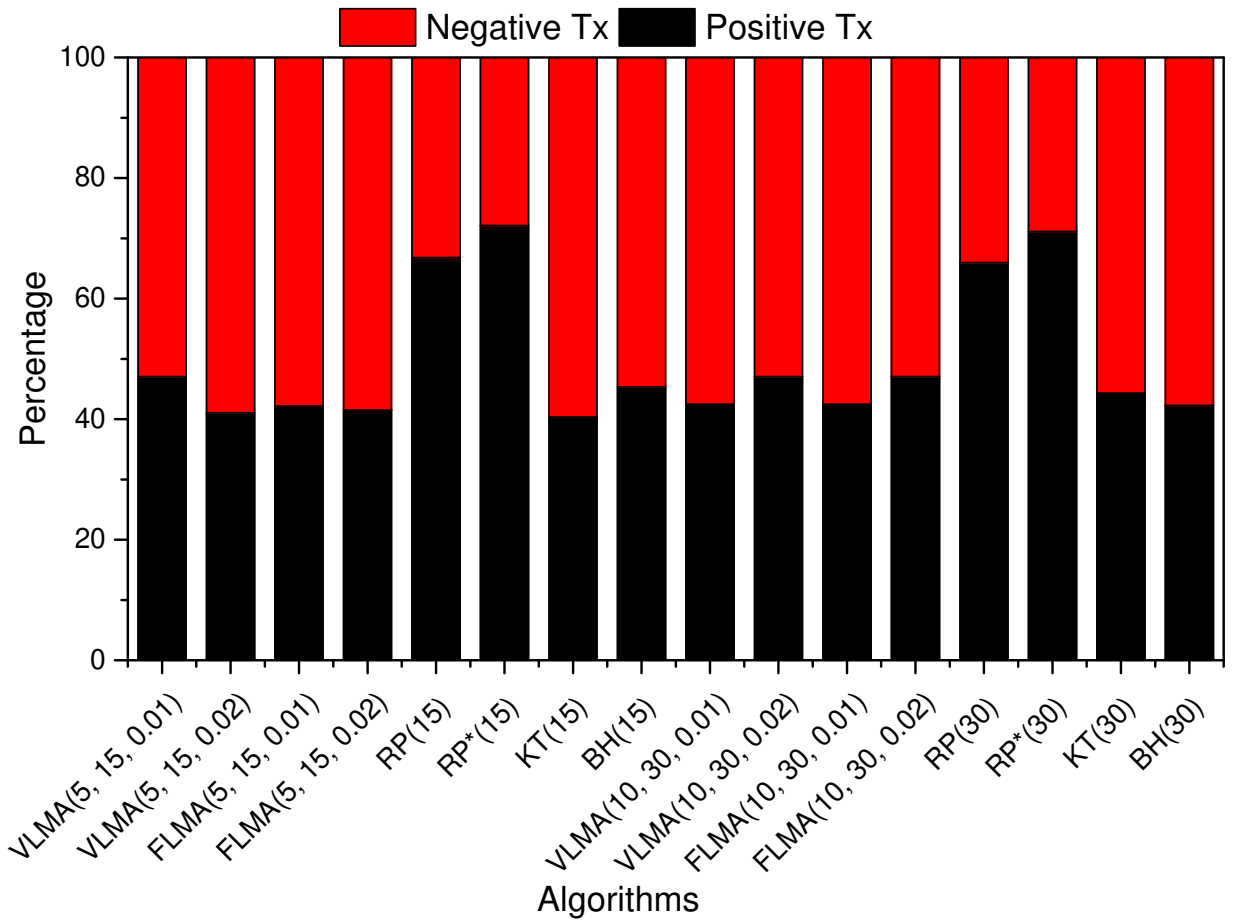

Figure 2. Positive vs Negative Returned Transactions

\subsection{How the transaction fee effect the profitablity of algorithms?}

Transaction fee can be a vital factor in the profitability of any trading algorithm. We consider a transaction fee $T F=\{0 \%, 2 \%, 4 \%\}$ and calculate $G P R$ to find the effect on the profitability. Figure 3 is a graphical representation of the effect of transaction fee on GPR of algorithms for BTC. We observed an average 
reduction of 3.9\% and 7.6\% in the GPR of algorithms when transaction fee of $2 \%$ and $4 \%$ are levied. Introducing a transaction fee of $2 \%$ reduced the positive returned strategies from 11 to 5 only, which are further reduced to $2\left(R P\right.$ and $R P^{*}$ ) when the transaction fee is increased to $4 \%$. For Euro, the profitability of algorithm is severely reduced from 16 to 0 strategies when the transaction fee of $4 \%$ is introduced. Rather interestingly, for $Y e n$ the introduction of transaction fee reduces the number of profitable strategies from 15 to 4 after introduction of $2 \%$ transaction fee. However, there is no change in the number of profitable strategies when the transaction fee is increased to $4 \%$. For Yen, the 4 profitable strategies are $\operatorname{VLMA}(5,15,0.02), \operatorname{FLMA}(5,15,0.02), \operatorname{VLMA}(10,30,0.02), F L M A(10,30,0.02)$. This also reflects that for variable length moving average strategies to be profitable the band value is vital. For smaller band values, the strategies might not be profitable.

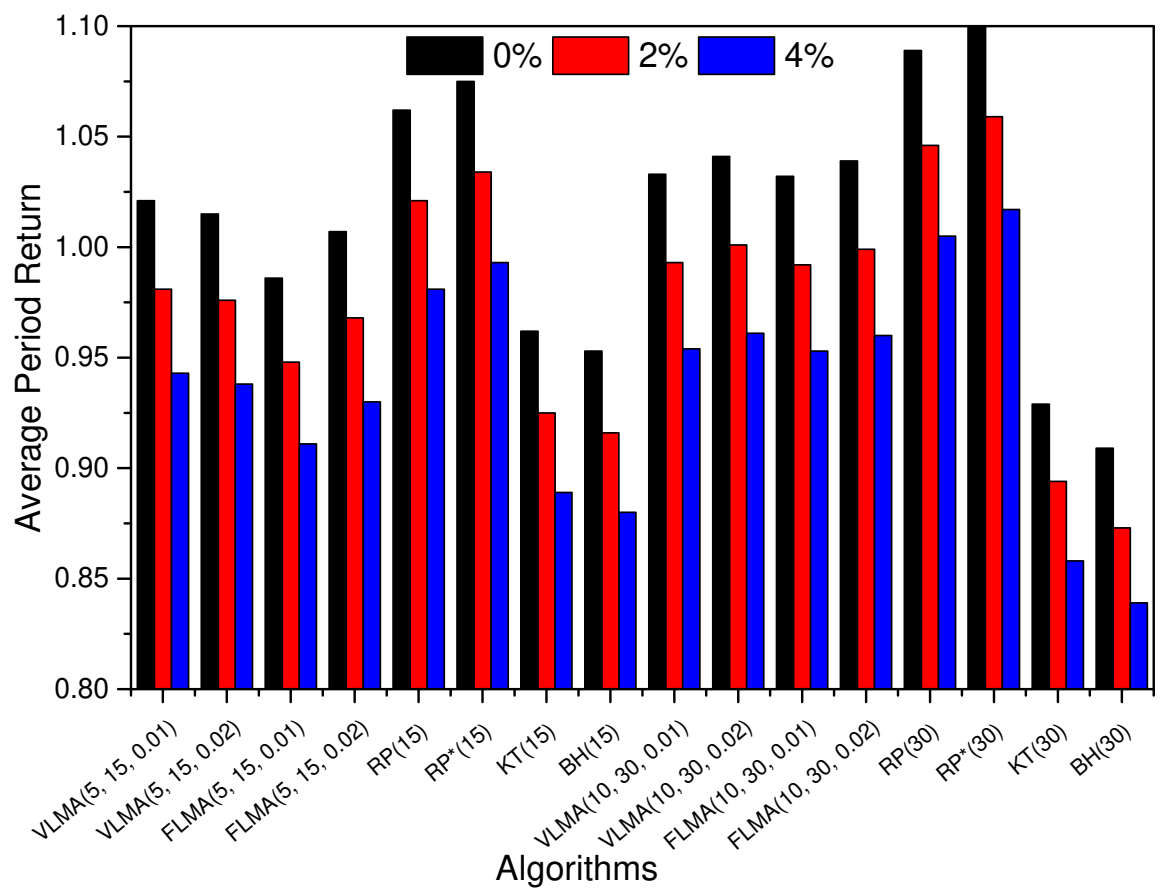

Figure 3. The effect of transaction fee on $G P R$ of $B T C$

\section{CONCLUSION}

We evaluated the short term profitability of $B T C$ over a set of reservation price and moving average based algorithms against Euro and Yen for a period of 8 years. Based on the average GPR, BTC seems less profitable venture than Yen, however, a deeper analysis revealed that the answer the profitability is strategy dependent as well. $R P^{*}$ achieved an average GPR of $10 \%$ for a trading period of 30 days, which is the maximum return obtained by any trading algorithm among the 3 assets. This confirmed BTC as an attractive investment opportunity for a short term investment. Our analysis also revealed that $R P$ and $R P^{*}$ are the best performing algorithms on $B T C$, whereas moving average based algorithms return higher profits for Euro and Yen. It is also shown that the selected set of algorithms beat the buy and hold approach except on Yen where the returns of $K T$ are less than that of buy and hold. Further, we highlighted that the returns of all the selected algorithms became negative except for $R P$ and $R P^{*}$ when a transaction fee of $2 \%$ was introduced. Increasing the transaction fee to $4 \%$ resulted in positive returns for $R P$ and $R P^{*}$ on 30 days investment horizon. For all other algorithms and their variants the returns were negative for a transaction fee of $4 \%$.

To the best of our knowledge, this study is the first of its kind to evaluate the profitability of BTC using a set of trading algorithms and against fiat currencies. Future work can include finding an optimized portfolio of fiat and crypto-currencies for short and long term investment. 


\section{REFERENCES}

Abbey, B. S. and Doukas, J. A. (2012). Is technical analysis profitable forindividual currency traders? The Journal of Portfolio Management, 39(1):142-150.

Ahmad, I. and Schmidt, G. (2012). An experimental analysis of online unidirectional conversion problem. In Huemer, C. and Lops, P., editors, E-Commerce and Web Technologies, pages 176-187, Berlin, Heidelberg. Springer Berlin Heidelberg.

Alessandretti, L., ElBahrawy, A., Aiello, L. M., and Baronchelli, A. (2018). Anticipating cryptocurrency prices using machine learning. Complexity, 2018.

Baur, D. G., Dichtl, H., Drobetz, W., and Wendt, V.-S. (2018). Investing in gold - market timing or buy-and-hold? International Review of Financial Analysis.

Brière, M., Oosterlinck, K., and Szafarz, A. (2015). Virtual currency, tangible return: Portfolio diversification with bitcoin. Journal of Asset Management, 16(6):365-373.

Brock, W., Lakonishok, J., and LeBaron, B. (1992). Simple technical trading rules and the stochastic properties of stock returns. The Journal of Finance, 47(5):1731-1764.

Chang, Y.-H., Jong, C.-C., and Wang, S.-C. (2017). Size, trading volume, and the profitability of technical trading. International Journal of Managerial Finance, 13(4):475-494.

Coakley, J., Marzano, M., and Nankervis, J. (2016). How profitable are fx technical trading rules? International Review of Financial Analysis, 45:273 - 282.

El-Yaniv, R., Fiat, A., Karp, R. M., and Turpin, G. (2001). Optimal search and one-way trading online algorithms. Algorithmica, 30:101-139.

Fang, J., Jacobsen, B., and Qin, Y. (2014). Predictability of the simple technical trading rules: An out-of-sample test. Review of Financial Economics, 23(1):30 - 45.

Gunasekarage, A. and Power, D. M. (2001). The profitability of moving average trading rules in south asian stock markets. Emerging Markets Review, 2(1):17 - 33.

Hsu, P.-H., Hsu, Y.-C., and Kuan, C.-M. (2010). Testing the predictive ability of technical analysis using a new stepwise test without data snooping bias. Journal of Empirical Finance, 17(3):471 - 484.

Hsu, P.-H., Taylor, M. P., and Wang, Z. (2016). Technical trading: Is it still beating the foreign exchange market? Journal of International Economics, 102:188 - 208.

Iqbal, J. and Ahmad, I. (2015). Optimal online k-min search. EURO Journal on Computational Optimization, 3(2):147-160.

Iqbal, J., Ahmad, I., and Schmidt, G. (2012). Can online trading algorithms beat the market? an experimental evaluation. In 3rd Student Conference on Operational Research. Schloss DagstuhlLeibniz-Zentrum fuer Informatik.

Iqbal, J., Ahmad, I., and Shah, A. (2019). Competitive algorithms for online conversion problem with interrelated prices. International Journal of Advanced Computer Science and Applications, 10(6).

Jiang, F., Tong, G., and Song, G. (2019). Technical analysis profitability without data snooping bias: Evidence from chinese stock market. International Review of Finance, 19(1):191-206.

Kao, M.-Y. and Tate, S. R. (1999). On-line difference maximization. SIAM Journal on Discrete Mathematics, 12(1):78-90.

Menkhoff, L. and Taylor, M. P. (2007). The obstinate passion of foreign exchange professionals: Technical analysis. Journal of Economic Literature, 45(4):936-972.

Mohr, E., Ahmad, I., and Schmidt, G. (2014). Online algorithms for conversion problems: A survey. Surveys in Operations Research and Management Science, 19(2):87-104.

Moore, T. (2013). The promise and perils of digital currencies. International Journal of Critical Infrastructure Protection, 3(6):147-149.

Nakamoto, S. et al. (2008). Bitcoin: A peer-to-peer electronic cash system.

Narayanan, A., Bonneau, J., Felten, E., Miller, A., and Goldfeder, S. (2016). Bitcoin and Cryptocurrency Technologies: A Comprehensive Introduction. Princeton University Press, Princeton, NJ, USA.

Nguyen, T., de Bodisco, C., and Thaver, R. (2018). Factors affecting bitcoin price in the cryptocurrency market: An empirical study. International Journal of Business \& Economics Perspectives, 13(1).

Schmidt, G., Mohr, E., and Kersch, M. (2010). Experimental analysis of an online trading algorithm. Electronic Notes in Discrete Mathematics, 36:519-526.

Schroeder, P., Dochow, R., and Schmidt, G. (2018). Optimal solutions for the online time series search and one-way trading problem with interrelated prices and a profit function. Computers \& Industrial Engineering, 119:465 - 471. 
Strobel, M. and Auer, B. R. (2018). Does the predictive power of variable moving average rules vanish over time and can we explain such tendencies? International Review of Economics \& Finance, 53:168 184.

Uras, N., Marchesi, L., Marchesi, M., and Tonelli, R. (2020). Forecasting bitcoin closing price series using linear regression and neural networks models. PeerJ Computer Science, 6:e279.

Wang, S. and Vergne, J.-P. (2017). Buzz factor or innovation potential: What explains cryptocurrencies' returns? PloS one, 12(1):e0169556.

Żbikowski, K. (2016). Application of Machine Learning Algorithms for Bitcoin Automated Trading, pages 161-168. Springer International Publishing, Cham.

Zhu, H., Jiang, Z.-Q., Li, S.-P., and Zhou, W.-X. (2015). Profitability of simple technical trading rules of chinese stock exchange indexes. Physica A: Statistical Mechanics and its Applications, 439:75 - 84. 